Research Article

\title{
Modified Four-Point Scleral Suture Fixation Technique for Repositioning a Dislocated Intraocular Lens in the Absence of Capsule Support
}

\author{
Kaicheng Wu, ${ }^{1,2}$ Wangyi Fang, ${ }^{1,2}$ Yuan Zong, ${ }^{1,2}$ Jian Yu, ${ }^{1,2}$ Canqing Xu, ${ }^{1,2}$ \\ Chunhui Jiang $\mathbb{1},{ }^{1,2}$ and Ye Tan $\mathbb{1}^{3}$ \\ ${ }^{1}$ Department of Ophthalmology and Vision Science Eye and ENT Hospital, Fudan University, Shanghai 200031, China \\ ${ }^{2}$ Key Laboratory of Myopia of State Health Ministry, Key Laboratory of Visual Impairment and Restoration of Shanghai, \\ Shanghai 200031, China \\ ${ }^{3}$ Gongli Hospital of Shanghai Pudong New Area, Shanghai 200135, China
}

Correspondence should be addressed to Chunhui Jiang; chhjiang70@163.com and Ye Tan; tan.ye@live.cn

Received 2 September 2020; Accepted 20 September 2020; Published 19 November 2020

Academic Editor: Zisis Gatzioufas

Copyright $(2020$ Kaicheng Wu et al. This is an open access article distributed under the Creative Commons Attribution License, which permits unrestricted use, distribution, and reproduction in any medium, provided the original work is properly cited.

Purpose. To study the efficacy of a modified four-point fixation technique for the repositioning of a dislocated intraocular lens (IOL) with four eyelets in the absence of capsule support. Methods. Four patients with dislocated four-eyelet hydrophilic acrylic IOLs (Akreos AO60) were enrolled. The modified technique combined four-point fixation with intrascleral sutures and suture burying. The technique minimized the limbus incision to $1 \mathrm{~mm}$ with no externalization of the IOL or its haptics. Follow-ups included routine ophthalmic examinations, corneal endothelial cell counts, and measurement of IOL tilt and decentration (measured using Pentacam ${ }^{\circledR}$ HR images). Results. The IOLs were successfully repositioned in all cases. After a mean follow-up period of $19.75 \pm 7.85$ months (range: 8 to 24 months), the patients' best-corrected vision acuity (BCVA (LogMAR), before: $0.63 \pm 0.36$, after: $0.58 \pm 0.43, P=0.604$ ) and intraocular pressure (pre $13.35 \pm 0.85 \mathrm{mmHg}$, post $14.80 \pm 2.03 \mathrm{mmHg}, P=0.150$ ) remained unchanged. Corneal endothelium density decreased about $6.84 \pm 2.97 \%$. In all cases, the IOL was well positioned during the follow-up. At the final visit, the average IOL tilt was $1.36 \pm 0.35^{\circ}$ horizontally and $1.31 \pm 0.14^{\circ}$ vertically. The average IOL decentration was $0.23 \pm 0.12 \mathrm{~mm}$ horizontally and $0.18 \pm 0.13 \mathrm{~mm}$ vertically. Conclusions. With this modified technique, dislocated IOLs with four-eyelets could be treated safely with favorable outcomes.

\section{Introduction}

Intraocular lens (IOL) dislocation has become increasingly problematic; the incidence of IOL dislocation is reported to be 0.2 to $3.0 \%$ [1]. Techniques to manage dislocation include IOL exchange and IOL repositioning [2]. Exchange techniques usually require a larger incision to extract the IOL, subsequently increasing the risk of significant astigmatism, vitreous prolapse, and intraocular bleeding [3]. Repositioning is beneficial as there is reduced postoperative astigmatism and other complications. Among all techniques used for IOL repositioning, the sutured scleral-fixed technique continues to be widely used [4]. With the sutured scleral-fixed technique, the IOL is placed in the correct anatomic position, thereby reducing the number of optical aberrations and decreasing the rate of secondary glaucoma, pigment dispersion, and abnormal pupillary movement. But in geometry, two points only define a line, whereas three points are required to define a plane. And, two-point scleral fixation has been reported to have less favourable IOL positioning [5]. In contrast, the Akreos IOL has four eyelets in haptics [6], which allow for four-point fixation to minimize IOL tilt and decentration. Here, we describe a modified four-point scleral fixation technique, which also combined intrascleral suture and suture burying, for the repositioning of dislocated four-eyelet IOLs. 


\section{Materials and Methods}

This study was approved by the Ethics Committee of the Eye and ENT Hospital of Fudan University and adhered to the Declaration of Helsinki. Informed consent was obtained from each patient.

Four patients diagnosed with Akreos AO60 IOL (Baush and Lomb, Inc.) dislocation were consecutively enrolled and treated by a single retina specialist (CH. J.) at the Eye and ENT Hospital, Fudan University, between May 2018 and November 2019.

The patients underwent a thorough ophthalmic examination before the operation, and the following measurements were collected: best-corrected visual acuity (BCVA); intraocular pressure (IOP) using a noncontact tonometer; spherical equivalent (SE), calculated as one-half of the cylindrical dioptric (C) plus the spherical diopter (D) power; corneal endothelium count using a noncontact specular microscope (Topcon America Corporation, Paramus, NJ, USA); and axial length (AL) using an IOLmaster (version 3.01; Carl Zeiss Meditec, Jena, Germany). Postoperatively, the patients were examined 1 day, 1 week, 1 month, 3 months, 6 months, 1 year, and 2 years later, and the following data were recorded: slit-lamp microscopy examination findings, BCVA, SE, IOP, and corneal endothelial cell density. IOL tilt and decentration were measured using anterior segment tomography with a Pentacam HR (Oculus Optikgeräte GmbH, Wetzlar, Germany) [7, 8].

2.1. Statistical Analysis. Statistical analyses were performed using SPSS Statistics (Version 20.0, IBM Corp., Armonk, NY, USA). Paired $t$ tests were used to compare the differences in BCVA, SE, IOP, and ECD pre- and postoperation. One-way analysis of variance was used to compare IOL tilt and decentration at 1 month, 6 months, and 2 years after surgery, followed by a least significant difference test for multiple comparisons. A level of $P<0.05$ was considered statistically significant.

2.2. Surgical Technique. After pupil dilation and retrobulbar block anesthesia, standard 23-G pars plana vitrectomy (PPV) was performed, except for one patient who had previously undergone vitrectomy. All dislocated IOLs were checked and were found to be hydrophilic acrylic IOLs (Akreos AO60, Bausch and Lomb Inc., Rochester, NY, USA) (Figure 1(a)). Two conjunctival snips were made at the nasal and temporal. Two limbus incisions were created at the nasal and temporal limbi with an angled (15 degree) blade. Using two 23-G vitreous forceps and the hand-shaking technique or by making it float to the posterior chamber using perfluorocarbon liquid (PFCL), the IOL was brought to the posterior chamber and incarcerated into the pupil, with one eyelet stretching into the anterior chamber. The anterior chamber was filled with viscoelastic material (DisCoVisc; Alcon Laboratories, Inc., Fort Worth, TX, USA). Two scleral incisions were made at 3 and 9 o'clock, $2 \mathrm{~mm}$ posterior to the limbus, with a 23-G MVR knife. Thereafter, a CIF-4 needle with a double 10-0 polypropylene (Prolene ${ }^{\circledR}$ ) suture passed through a partial thickness of the sclera, entered at the incision at 9 o'clock, and exited at 7 o'clock (Figure 1(b)). A rather long suture tail was left at the 9 o'clock position. The needle was then inserted into the posterior chamber at the exit position; then, using $23-\mathrm{G}$ vitreous forceps to hold the IOL, the needle was passed through the nasal inferior eyelet and exited the eye through the limbus at the 1 o'clock position (Figure 1(c)). The needle was then removed from the suture, and the suture was reintroduced into the anterior chamber, passed through the nasal superior eyelet, and exited the eye through the scleral incision at 9 o'clock, with the help of two 23-G vitreous forceps (Figures 1(d) and 1(e)).

On the temporal side, we adopted another maneuver. After the needle was passed through the sclera from 3 o'clock to 1 o'clock, it was introduced into the posterior chamber and passed through the temporal superior eyelet. Using the guidance of a 23-G vitreous forceps, the needle exited the eye through the nasal limbus incision that had previously been created (Figure 1(f)). Then, through the same incision, the needle was reintroduced into the anterior chamber, passed through the temporal inferior eyelet and exited the eye through the scleral incision at 3 o'clock with the help of 23-G vitreous forceps (Figure $1(\mathrm{~g})$; the two different maneuvers are presented in Video 1). At this point, the two ends of the suture were tied on both sides, and care was taken to centralize the IOL. The suture with the CIF-4 needle was tied to the other tail or tails of the knot that were without a needle (Figure 1(h)) and was passed through the partial thickness of the sclera twice, using a technique similar to the Z-suture technique described by Szurman et al. [9]. Then, after a thorough check of the fundus, the PFCL was removed (if used) and the scleral and conjunctival incisions were closed with 8-0 sutures (Figure 1(i)). Video demonstrating the surgical procedure was available at https://drive.google.com/ file/d/1ABxiA9scqA-DbO63JoIWdMYxAZfTlgx4/view.

\section{Results}

Four patients (mean age: $59.75 \pm 13.72$ years) were enrolled. The average axial length was $28.97 \pm 4.53 \mathrm{~mm}$ (range: 24.84-33.33 mm), and IOL dislocation occurred an average of $8.5 \pm 1.29$ (range: $7-10$ ) years from the time of cataract surgery. On average, the operations took $41.00 \pm 9.42$ minutes and were completed without severe complications, except for mild hemorrhage at the site of scleral incision in one case (Table 1).

IOP was elevated in case 1 at the 1 -month follow-up. Antiglaucoma medication (brimonidine tartrate eye drops ${ }^{\circledR}$ 0.15\%; Alphagan ${ }^{\circledR}$ P.0. 15\%; Allergan, Inc., Irvine, CA, USA) was used and gradually withdrawn over 3 weeks. Case 2 suffered from transitory elevated IOP with hyphemia 3 days after surgery. With paracentesis and topical antiglaucoma agents, the IOP returned to normal; at the 1-month followup, the patient did not require any antiglaucoma medication. The IOP was then maintained and remained within a normal range in these two patients throughout the 2-year follow-up period without antiglaucoma medication. No other postoperative complications were noticed during the follow-up period. 

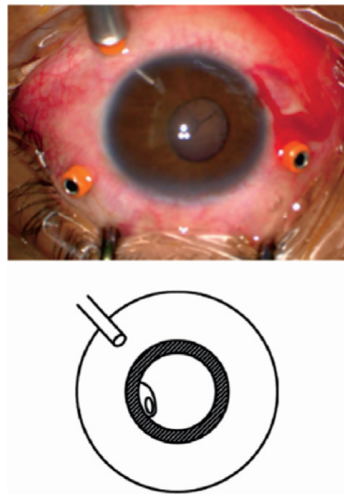

(a)
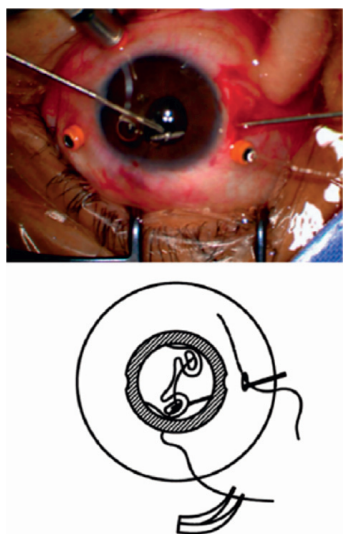

(d)
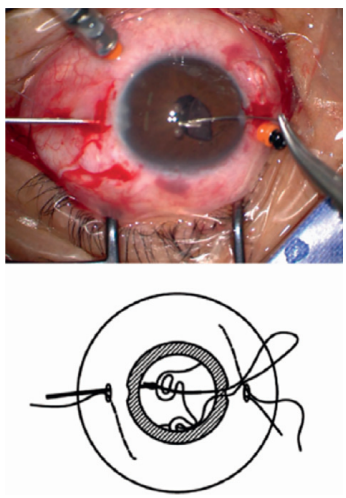

(g)
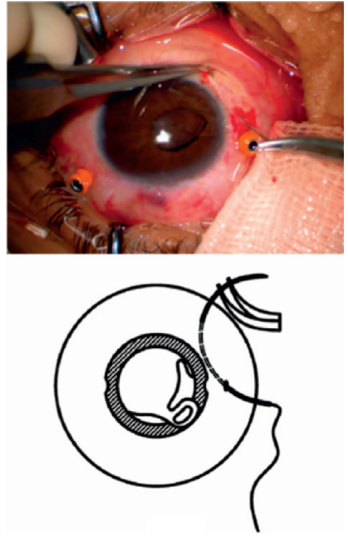

(b)
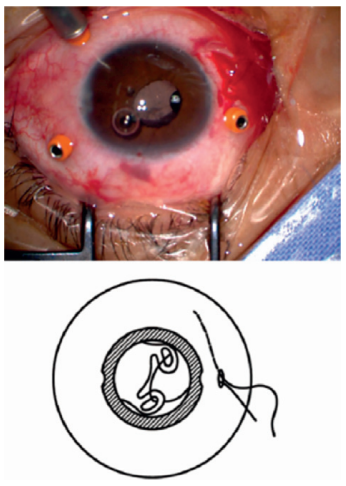

(e)
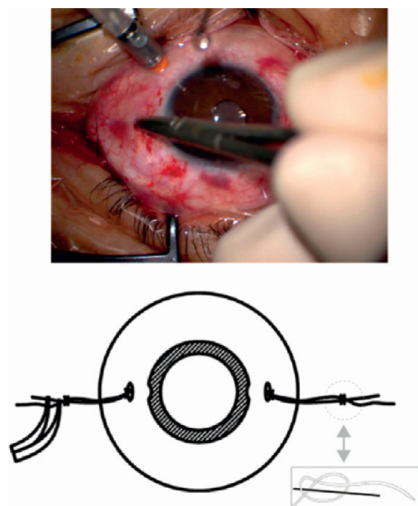

(h)
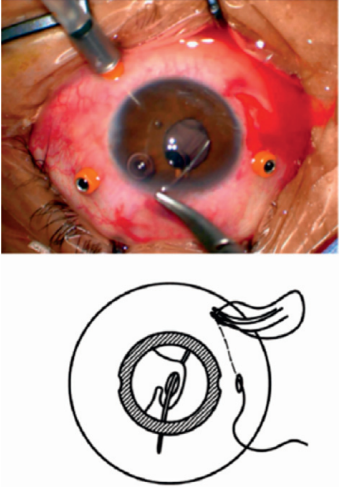

(c)
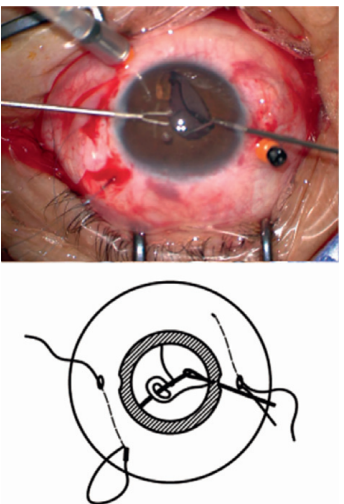

(f)
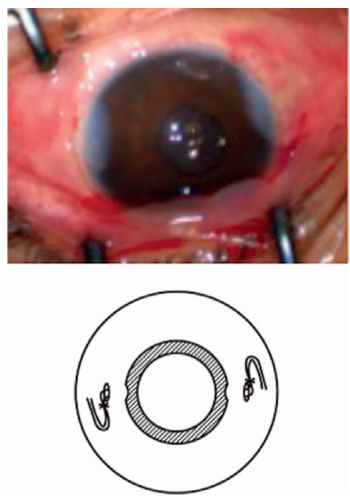

(i)

FIGURE 1: Surgical steps. (a) A dislocated intraocular lens (IOL). (b) After the IOL was brought to the posterior chamber, a CIF-4 needle with a double 10-0 polypropylene (Prolene ${ }^{\circledR}$ ) suture was passed through the partial thickness of the sclera at the incision from the 9 o'clock to the 7 o'clock position. (c) The needle was inserted into the posterior chamber through the sclera at the 7 o'clock position and was passed through the nasal inferior eyelet and then externalized through the limbus at the 1 o'clock position. (d) The needle was then removed, and the suture was reintroduced into the anterior chamber and passed through the nasal superior eyelet using vitreous forceps. (e) Using these vitreous forceps, the suture was then externalized through the scleral incision at 9 o'clock. (f) On the temporal side, after passing through the sclera from 3 o'clock to 1 o'clock, the needle was introduced into the posterior chamber, passed through the temporal superior eyelet, and externalized through the nasal limbus incision with the help of vitreous forceps. (g) Through the same incision, the needle was reintroduced into the anterior chamber, passed through the temporal inferior eyelet, and externalized through the scleral incision at 3 o'clock using vitreous forceps. (h) The two ends of the suture, on both sides, were tied and care was taken to centralize the IOL. Then, the suture with a CIF-4 needle was tied to the other suture tail (or tails lacking a needle) using a sliding knot (shown in the small diagram). (i) The sutures were passed through the partial thickness of the sclera, twice, using a technique similar to the Z-suture technique described by Szurman et al. 
TABLE 1: Individual patient characteristics.

\begin{tabular}{|c|c|c|c|c|c|c|c|}
\hline Case & $\begin{array}{c}\text { Sex }(F / M) / \text { age } \\
\text { (years)/eye }(\mathrm{R} / \mathrm{L})\end{array}$ & $\begin{array}{l}\text { Axial } \\
\text { length } \\
(\mathrm{mm})\end{array}$ & Other ocular disease & $\begin{array}{c}\text { IOL } \\
\text { implantation to } \\
\text { repositioning } \\
\text { (years) }\end{array}$ & $\begin{array}{l}\text { Risk factor(s) for } \\
\text { zonular defect }\end{array}$ & $\begin{array}{l}\text { Operation } \\
\text { time } \\
\text { (minutes) }\end{array}$ & $\begin{array}{l}\text { Follow-up } \\
\text { (months) }\end{array}$ \\
\hline 1 & $\mathrm{M} / 40 / \mathrm{L}$ & 25.28 & None & 8 & Unknown & 48 & 24 \\
\hline 2 & $\mathrm{~F} / 68 / \mathrm{L}$ & 32.41 & High myopia & 10 & Unknown & 35 & 24 \\
\hline 3 & $\mathrm{M} / 70 / \mathrm{R}$ & 24.84 & $\begin{array}{l}\text { Rhegmatogenous retinal } \\
\text { detachment ( } 2 \text { years ago) }\end{array}$ & 7 & $\begin{array}{l}\text { PPV + silicone oil } \\
\text { tamponade }\end{array}$ & 30 & 23 \\
\hline 4 & $\mathrm{~F} / 61 / \mathrm{L}$ & 33.33 & Uveitis optic atrophy & 9 & Unknown & 50 & 8 \\
\hline
\end{tabular}

F, female; M, male; R, right; L, left.

After the surgery, bare vision improved rapidly in all cases. The IOLs were found to be well-centered and remained stable throughout the follow-up period. After an average follow-up period of $19.75 \pm 7.84$ (range: 8-24) months, the BCVA (LogMAR, pre $0.63 \pm 0.36$, post $0.58 \pm 0.43, P=0.604$ ) and IOP (pre $13.35 \pm 0.85 \mathrm{mmHg}$, post $14.80 \pm 2.03 \mathrm{mmHg}, P=0.150)$ remained unchanged. The average corneal endothelial cell density decreased from $2344.50 \pm 441.24$ cells $/ \mathrm{mm}^{2}$ to $2192 \pm 465.95 \mathrm{cells} / \mathrm{mm}^{2}$ $(P=0.009)$; average decrease was $6.84 \pm 2.97 \%$ (Table 2$)$.

At the last follow-up visit, the average IOL tilts were $1.36 \pm 0.35^{\circ}$ horizontally and $1.31 \pm 0.14^{\circ}$ vertically. The average IOL decentration was $0.23 \pm 0.12 \mathrm{~mm}$ horizontally and $0.18 \pm 0.13 \mathrm{~mm}$ in vertically. In three cases with more than 1 year follow-up (Table 3 ), the IOL position remained stable throughout the 1-month, 6-month, and 2-year follow-up periods (Table 4).

\section{Discussion}

Posterior chamber IOL (PCIOL) dislocation is one of the most serious complications following phacoemulsification. Management includes IOL exchange or IOL repositioning [4]. Repositioning is beneficial as it only requires a small incision, thus reducing the risk of additional endothelial cell trauma and postoperative astigmatism [2]. Here, we presented a technique that combined four-point scleral fixation, the intrascleral suture technique, and suture burying to reposition dislocated four-eyelet hydrophilic acrylic IOLs (Akreos AO60, Bausch and Lomb Inc., Rochester, NY, USA). The main purpose of this new technique is to minimize the incision as well as IOL tilt and decentration. Our primary results suggested that, with incisions $<1 \mathrm{~mm}$, the modified technique achieved good IOL positioning.

The modified technique ensures that the incision is minimized. Despite improved techniques which are currently used, extracting an IOL [10] or eternalizing the haptics $[11,12]$ would induce a larger wound. However, our technique minimized incisions to less than $1 \mathrm{~mm}$, as only side ports and 23-G scleral incisions were made. Various studies have demonstrated that a smaller incision size is beneficial for (1) reducing surgical astigmatism [13-15], (2) rapid wound healing, (3) decreasing the risk of endophthalmitis, and (4) incurring fewer intraoperative complications. With a minimized incision, astigmatism remained unchanged in all four cases in this study (Table 2). Moreover, the endothelial cell density loss (ECL), an important indicator of surgical safety, was $6.84 \pm 2.97 \%$ in our study, close to the ECL following an uneventful phacosurgery [16].

It has previously been reported that IOL tilt and decentration with two-point scleral fixation are much higher than that with in-the-bag IOL implantation (two-point scleral vs in-the-bag: tilt $6.0^{\circ}$ vs $1.5^{\circ}$, decentration $0.6 \mathrm{~mm}$ vs $0.3 \mathrm{~mm}$ ) [5, 17-20] (Supplemental Table 1). Further, an in vitro experiment demonstrated that it is very difficult to avoid tilt and decentration if two-point scleral fixation is used [21]. In 2009, Oren first reported four-point Akeros AO60 IOL scleral fixation for IOL implantation without sufficient capsular support, and their results were encouraging [22]. To the best of our knowledge, no previous study has directly compared IOL positioning between two- and four-point scleral fixation. In our study, the average H-IOL tilt was $1.36 \pm 0.35^{\circ}$ and $\mathrm{V}$-IOL tilt was $1.31 \pm 0.14^{\circ}$, while the average $\mathrm{H}-\mathrm{IOL}$ decentration was $0.23 \pm 0.12 \mathrm{~mm}$ and $\mathrm{H}-\mathrm{IOL}$ decentration was $0.18 \pm 0.13 \mathrm{~mm}$. These results were better than those obtained with two-point fixation [17] and were comparable to the results after uneventful phacoemulsification [18].

Another technique used in our approach was intrascleral sutures, which negated the need for a scleral flap or groove. Compared to the flap and groove technique, the intrascleral suture is easier to make, and if the needle does not exit at the ideal location (i.e., $2 \mathrm{~mm}$ from the limbus and $4-5 \mathrm{~mm}$ from the scleral incision), the needle can simply be withdrawn and another attempt can be made. Additionally, the position of the first set of sutures is directly visible, which makes it easier to place the second set of sutures exactly $180^{\circ}$ opposite to the first set. In addition, at all four points, the suture was introduced through the sclera using an ab externo technique; thus, it was easy to ensure that all four points were at the same distance from the limbus and in the same plane [23]. Consequently, IOL tilt should be greatly reduced.

Moreover, we made use of the intrascleral burying technique to protect the suture knot. After making the suture knot, instead of cutting the suture and rotating it, we tied the double 10-0 polypropylene (Prolene ${ }^{\circledR}$ ) with a CIF-4 needle to the tail or tails of the knot and buried the tails into the sclera. The main purpose of this step was to reduce the friction between the suture tails and the sclera or conjunctiva, which was the main reason for erosion and exposure. The rationale behind this was that, once the suture is cut, the short cut-ends are quite stiff and may erode the 


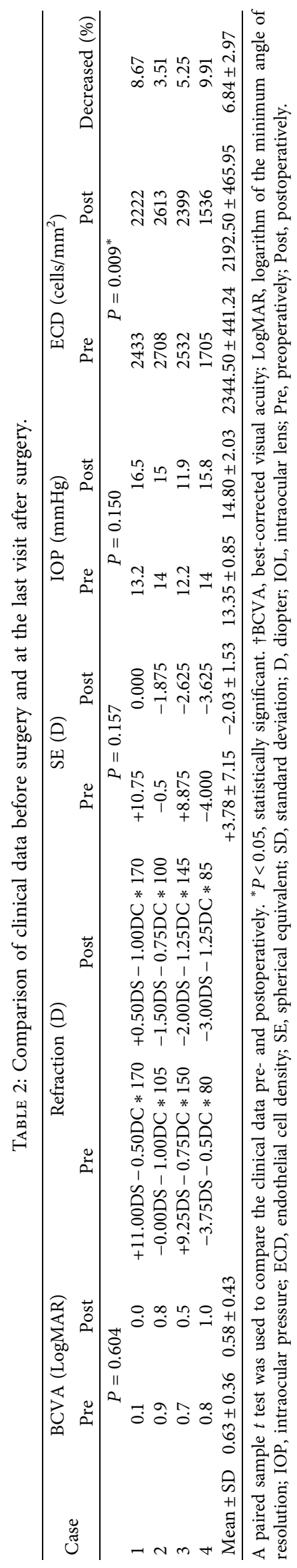




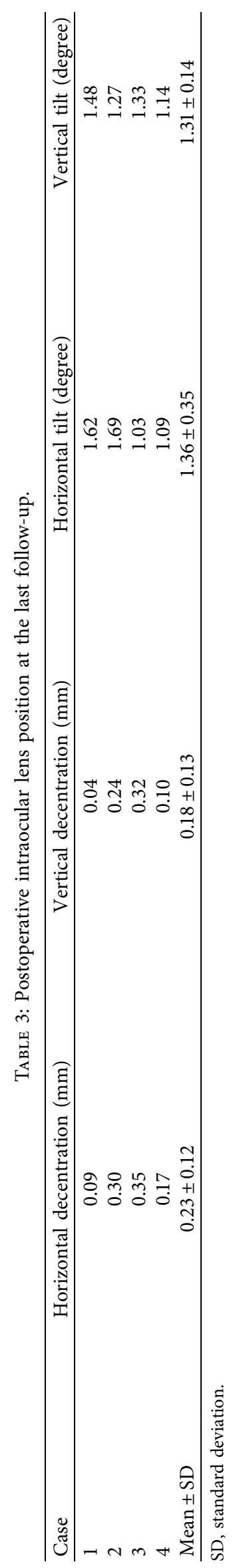




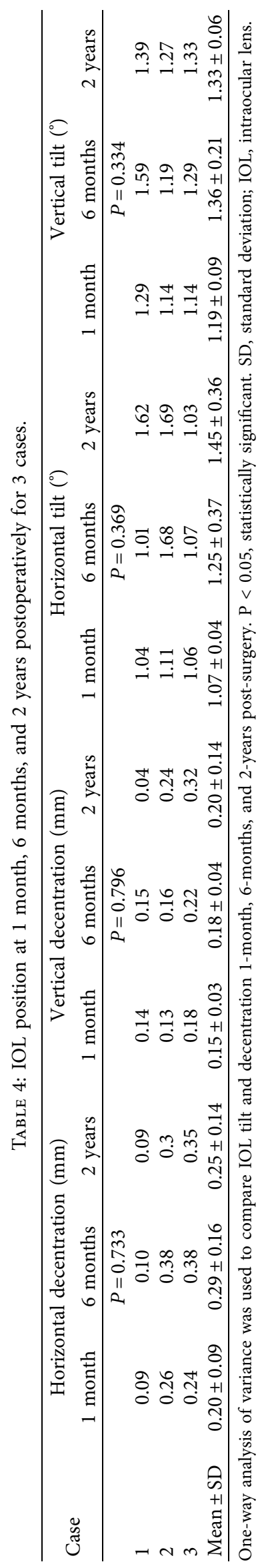


sclera [24]; however, as the tails used in our approach were much longer, they should have better pliability and lie tangential to the sclera and thus should stay within the sclera without creating much friction.

\section{Conclusions}

The number of cases and follow-up period were limited, but the primary data suggested that with this new technique, dislocated four-eyelet IOLs could be repositioned successfully with reduced surgical trauma, and good IOL positioning.

\section{Data Availability}

The data used to support the findings of this study are included within the article.

\section{Conflicts of Interest}

The authors declare that there are no conflicts of interest regarding the publication of this article.

\section{Authors' Contributions}

Kaicheng Wu and Wangyi Fang contributed equally to the work.

\section{Acknowledgments}

The publication of this article was supported in part by research grants from the Shanghai Committee of Science and Technology (grant nos. 19441900900 and 201409006800). The authors thank the subjects and our colleagues who helped perform this study.

\section{Supplementary Materials}

Supplemental Digital Content 1: video demonstrating the surgical procedure (MP4) ; Supplemental Table 1: comparison of the IOL tilt and decentration in four-point scleral suture fixation, two-point scleral suture fixation, and phacoemulsification. (Supplementary Materials)

\section{References}

[1] F. J. Ascaso, V. Huerva, and A. Grzybowski, "Epidemiology, etiology, and prevention of late IOL-capsular bag complex dislocation: review of the literature," Journal of Ophthalmology, vol. 2015, Article ID 805706, 2015.

[2] R. Sarrafizadeh, A. J. Ruby, T. S. Hassan et al., "A comparison of visual results and complications in eyes with posterior chamber intraocular lens dislocation treated with pars plana vitrectomy and lens repositioning or lens exchange," Ophthalmology, vol. 108, no. 1, pp. 82-89, 2001.

[3] E. C. Davies and R. Pineda, "Intraocular lens exchange surgery at a tertiary referral center: indications, complications, and visual outcomes," Journal of Cataract \& Refractive Surgery, vol. 42, no. 9, pp. 1262-1267, 2016.

[4] K. P. Dajee, A. M. Abbey, and G. A. Williams, "Management of dislocated intraocular lenses in eyes with insufficient capsular support," Current Opinion in Ophthalmology, vol. 27, no. 3, pp. 191-195, 2016.

[5] K. Hayashi, H. Hayashi, F. Nakao, and F. Hayashi, "Intraocular lens tilt and decentration, anterior chamber depth, and refractive error after trans-scleral suture fixation surgery11The authors have no proprietary interest in any of the materials described in this article," Ophthalmology, vol. 106, no. 5, pp. 878-882, 1999.

[6] D. C. Terveen, N. R. Fram, B. Ayres, and J. P. Berdahl, "Smallincision 4-point scleral suture fixation of a foldable hydrophilic acrylic intraocular lens in the absence of capsule support," Journal of Cataract \& Refractive Surgery, vol. 42, no. 2, pp. 211-216, 2016.

[7] K. Sasaki, Y. Sakamoto, T. Shibata, H. Nakaizumi, and Y. Emori, "Measurement of postoperative intraocular lens tilting and decentration using Scheimpflug images," Journal of Cataract \& Refractive Surgery, vol. 15, no. 4, pp. 454-457, 1989.

[8] X. Zhu, Y. Zhang, W. He et al., "Tilt, decentration, and internal higher-order aberrations of sutured posterior-chamber intraocular lenses in patients with open globe injuries," Journal of Ophthalmology, vol. 2017, Article ID 3517461, 2017.

[9] P. Szurman, k. Petermeier, S. Aisenbrey, M.S. Spitzer, G.B. Jaissle et al., "Z-suture: a new knotless technique for transscleral suture fixation of intraocular implants," British Journal of Ophthalmology, vol. 94, no. 2, pp. 167-169, Article ID $162180,2010$.

[10] T. John, S. Tighe, O. Hashem, and H. Sheha, "New use of 8-0 polypropylene suture for four-point scleral fixation of secondary intraocular lenses," Journal of Cataract \& Refractive Surgery, vol. 44, no. 12, pp. 1421-1425, 2018.

[11] A. I. Moawad and A. A. Ghanem, "One-haptic fixation of posterior chamber intraocular lenses without scleral flaps," Journal of Ophthalmology, vol. 2012, Article ID 891839, 2012.

[12] Y. Chantarasorn, S. Techalertsuwan, P. Siripanthong, and A. Tamerug, "Reinforced scleral fixation of foldable intraocular lens by double sutures: comparison with intrascleral intraocular lens fixation," Japanese Journal of Ophthalmology, vol. 62, no. 3, pp. 365-372, 2018.

[13] J. Wang, E.-K. Zhang, W.-Y. Fan, J.-X. Ma, and P.-F. Zhao, "The effect of micro-incision and small-incision coaxial phaco-emulsification on corneal astigmatism," Clinical \& Experimental Ophthalmology, vol. 37, no. 7, pp. 664-669, 2009.

[14] J. Yang, X. Wang, H. Zhang, Y. Pang, and R. H. Wei, “Clinical evaluation of surgery-induced astigmatism in cataract surgery using $2.2 \mathrm{~mm}$ or $1.8 \mathrm{~mm}$ clear corneal micro-incisions," International Journal of Ophthalmology, vol. 10, no. 1, pp. 68-71, 2017.

[15] S. C. Moon, T. Mohamed, and I. H. Fine, "Comparison of surgically induced astigmatisms after clear corneal incisions of different sizes," Korean Journal of Ophthalmology, vol. 21, no. 1, pp. 1-5, 2007.

[16] J. W. Ho and N. A. Afshari, "Advances in cataract surgery," Current Opinion in Ophthalmology, vol. 26, no. 1, pp. 22-27, 2015.

[17] A. Durak, H. F. Oner, N. Koçak, and S. Kaynak, "Tilt and decentration after primary and secondary transsclerally sutured posterior chamber intraocular lens implantation," Journal of Cataract \& Refractive Surgery, vol. 27, no. 2, pp. 227-232, 2001.

[18] M. M. Uzel, S. Ozates, M. Koc, A. G. Taslipinar Uzel, and P. Yılmazbaş, "Decentration and tilt of intraocular lens after posterior capsulotomy," Seminars in Ophthalmology, vol. 33, no. 6, pp. 766-771, 2018. 
[19] S. Ozates, M. Koc, M. M. Uzel, and P. Yilmazbas, "Comparison of intraocular lens position change following two different implantation techniques," Current Eye Research, vol. 42, no. 9, pp. 1235-1239, 2017.

[20] P. Rosales, A. De Castro, I. Jiménez-Alfaro, and S. Marcos, "Intraocular lens alignment from Purkinje and Scheimpflug imaging," Clinical and Experimental Optometry, vol. 93, no. 6, pp. $400-408,2010$.

[21] K. D. Teichmann and I. A. M. Teichmann, "The torque and tilt gamble," Journal of Cataract \& Refractive Surgery, vol. 23, no. 3, pp. 413-418, 1997.

[22] O. N. Fass and W. K. Herman, "Sutured intraocular lens placement in aphakic post-vitrectomy eyes via small-incision surgery," Journal of Cataract \& Refractive Surgery, vol. 35, no. 9, pp. 1492-1497, 2009.

[23] R. L. Bergren, "Four-point fixation technique for sutured posterior chamber intraocular lenses," Archives of Ophthalmology, vol. 112, no. 11, pp. 1485-1487, 1994.

[24] P. Oskala, "Friction knot to fixate scleral sutures," Journal of Cataract \& Refractive Surgery, vol. 41, no. 3, pp. 497-500, 2015. 